\title{
WAVE STRUCTURE WITHIN HI FILAMENTS AT HIGH GALACTIC LATITUDE AND THE NATURE OF "CLOUDS" IN INTERSTELLAR SPACE
}

\author{
GERRIT L. VERSCHUUR \\ 4802 Brookstone Terrace, Bowie, MD 20720
}

\begin{abstract}
Large amplitude waves have been found in the morphology and velocity patterns of several long filaments of $\mathrm{HI}$ at high latitude. $\mathrm{HI}$ in the filaments is controlled by magnetic fields and the velocity patterns and morphology bear the hallmarks of Alfvén waves. Enhanced emission features (EEFs), traditionally referred to as"clouds," are seen wherever a segment of flux tube is viewed end-on. This suggests that HI emission structure teaches us about field geometry and not about cloud physics. Similar effects have been recognized in other regions mapped with high-resolution as well as in completely mapped high-velocity "clouds."
\end{abstract}

\section{THE DATA}

Figure 1a shows a sketch of the filaments recognized in the data given by Verschuur (1974) for the region under consideration. Figure $1 \mathrm{~b}$ shows the velocity of gas associated with the major Filament $A$ as a function of position along its axis. $A$ first-order slope, part of a larger scale effect, was removed and the velocity residuals show a clear wave-like pattern, as is seen in Figure 1c. Comparison of these data with the morphology of the filament suggests a three dimensional wave structure, with amplitude $5 \mathrm{~km} / \mathrm{s}$ and $1 / 4 \lambda$. This is probably an Alfvén wave, consistent with a field of $5 \mu \mathrm{G}$ and a density of $5 \mathrm{~cm}^{-3}$.

Figure 2 is a detailed view of a sample of three channel maps compared with a schematic that shows the location of the filaments in a region of overlap. Enhanced emission features are indicated with labels giving RA, dec., and velocity. The curve labelled "simulation" is a projection of a helix viewed from a suitable angle to account for the filament that curves into and out of EEF H0936+00.0+11. Enhancement in 21-cm emission appears to be the result of viewing a segment of the helical flux tube end-on. The inset shows the area in which Guhathakurta and Tyson (1989) found optical emission associated with an enhancement of $100 \mu \mathrm{m}$ IR radiation. 


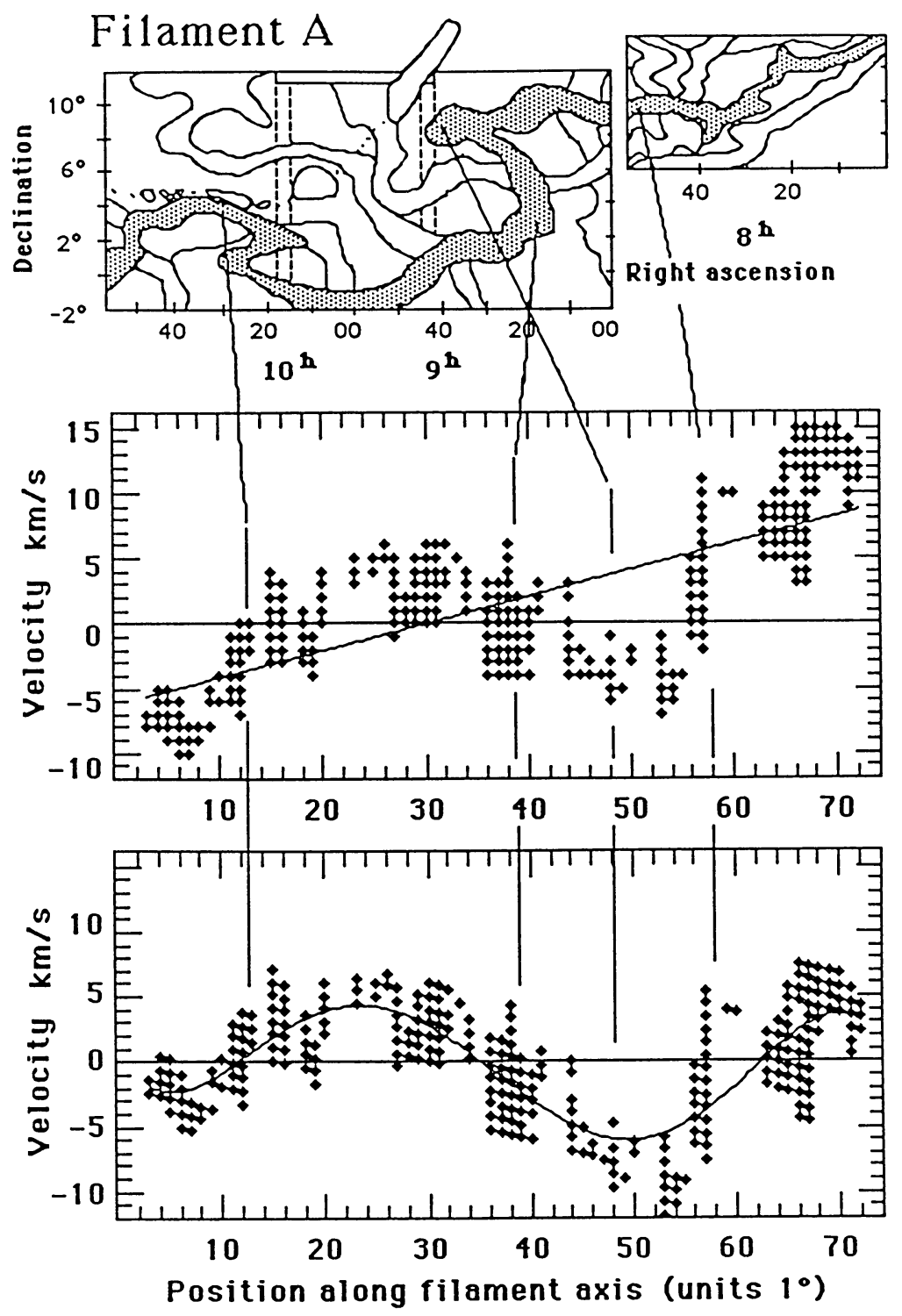

Figure 1. (a) Schematic of the filaments identified. The velocity of emission associated with Filament A (shaded) is shown in (b) After removal of a first-order slope, the velocity residuals (c) show a wave pattern that is closely related to the morphology of the filament. 

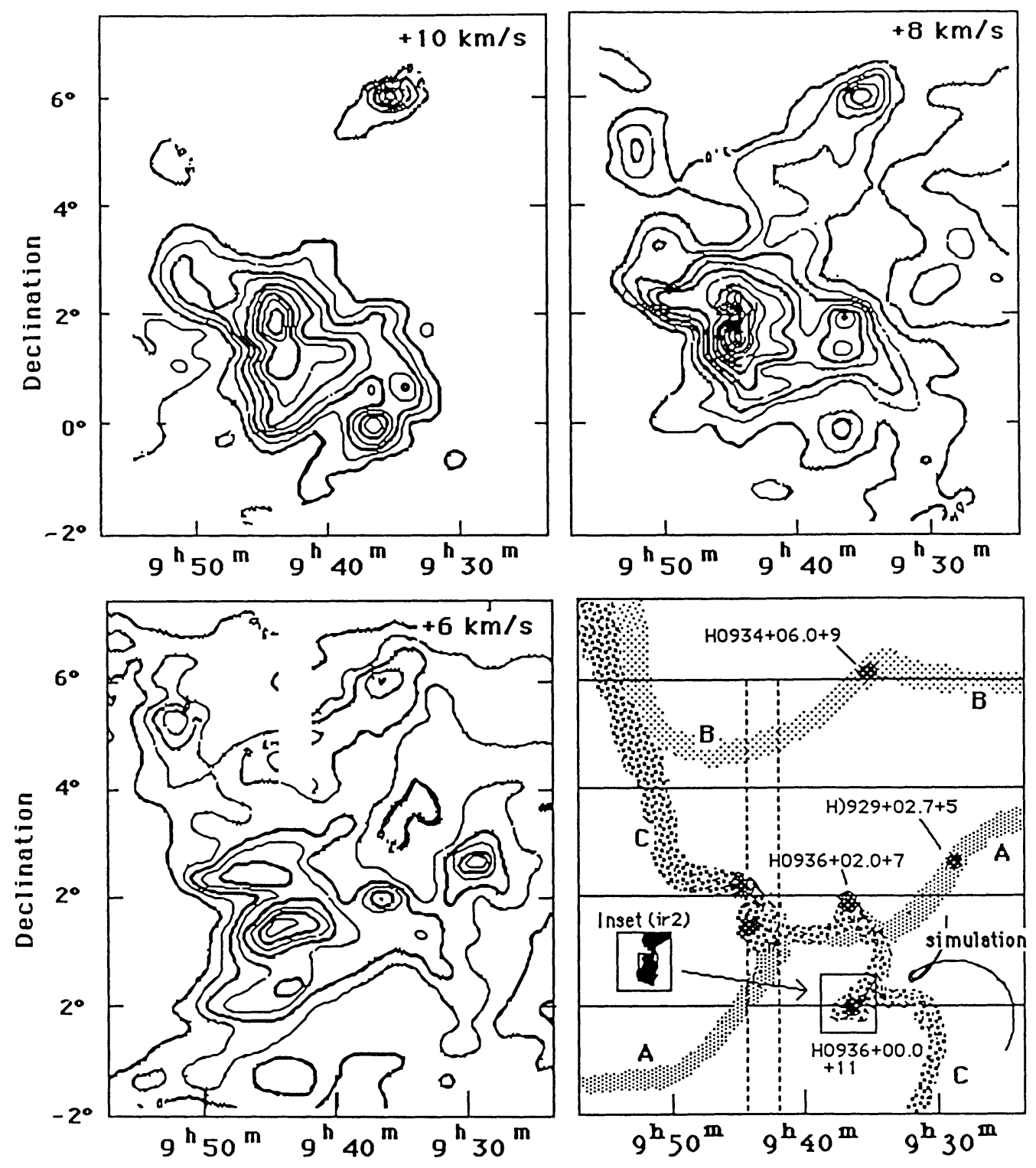

Right ascension

Figure 2. Three channel maps of a portion of area mapped (Figure 1a). Use of 29 channel maps allowed the filament pattern summarized in bottom-right diagram to be determined. The brightest EEFs occur where the filament axes show the most pronounced change in direction. The simulation was produced by rotating a helix and viewing it from an angle that gave good agreement with the pattern in the HI emission around $\mathrm{H} 0936+00.0+11$. 


\section{CONCLUSIONS}

The following statements summarize extensive analysis to be published elsewhere. Interstellar $\mathrm{HI}$ is found in filaments that twist in three dimensions. Gas motion is controlled by magnetic fields whose magnetic pressure (for $\mathrm{B}=5$ to $10 \mu \mathrm{G}$ ) controls thermal pressure of the $\mathrm{HI}$ (with density $5 \mathrm{~cm}^{-3}$ at $200 \mathrm{~K}$ or less). Wave structure of wavelength $30^{\circ}$ and amplitude $8^{\circ}$ in the morphology of these filament is associated with a velocity wave, amplitude $5 \mathrm{~km} / \mathrm{s}$. This is probably an Alfvén wave that is three dimensional. This pattern shows fine structure on several scale-lengths. Every enhanced emission feature ("cloud") in the region is part of one of seven filaments identified and is produced where the line-of-sight is along a local segment of twisted flux tube. EEFs thus teach us about field geometry and not about the physics of interstellar HI "clouds." Observations normal to a filament axis give column densities of $3 \times 10^{19} \mathrm{~cm}^{-2}$. The enhancement of emission in an EEF with respect to this value is an indicator of the depth of the segment of flux tube with respect to its width. These ratios are found to be 5 to 10 . Assumptions usually made regarding the depth of $\mathrm{HI}$ "clouds" being the same as their width may be seriously in error. Also, masses for nearly $400 \mathrm{HI}$ "clouds" found in the literature are probably incorrect. In the region studied, every enhancement in $100 \mu \mathrm{m}$ emission is associated either with a specific HI EEF and/or with the overlap of HI filaments in the line-of-sight. This suggests that cirrus structures at high latitudes may also be heavily modulated by geometric effects. Preliminary examination of other high-resolution HI data suggests that these phenomena are common. Structure at intermediate and high latitudes may be part of a single phenomenon involving a mass of twisted filaments, possibly remnants of an old supernova inside which the sun is located. Without exception, high-resolution HI data for high-velocity clouds show that they, too, are filamentary and within them EEFs occur where the line-ofsight is along a filament axis. Within such high-velocity filaments the structure is complex and preliminary interpretation of the data sugggests interesting constraints on the relative values of field strength and density in the HVCs. This, in turn, has a bearing on the peculiar phenomenon of the morphological similarity between $\mathrm{CO}$ clouds and HVCs reported by Verschuur (1990). Finally, it is noted that when a segment of a twisted HI filament is seen along the axis of the twist it will appear as a ring on the sky. Based on the data in Figure 1, such rings may have diameters as large as $10^{\circ}$. This phenomenon may be responsible for many of the apparent shell-like structures found in low resolution $21-\mathrm{cm}$ surveys.

\section{REFERENCES}

Guhathakurta, P. and Tyson, J. A. (1989) Ap. J. 346, 773

Verschuur, G. L. (1974) Ap. J. Suppl. 27, 65

Verschuur, G. L. (1990) Ap. J. September. In press. 